Federal Reserve Bank of Minneapolis

Research Department Staff Report 323

Revised September 2005

\title{
Opportunity and Social Mobility
}

Christopher Phelan*

Federal Reserve Bank of Minneapolis

ABSTRACT

This study argues that both unequal opportunity and social mobility are necessary implications of an efficient societal arrangement when incentives must be provided.

${ }^{*}$ The author thanks Marco Bassetto, V. V. Chari, Narayana Kocherlakota, Aleh Tsyvinski, and Ivan Werning for helpful comments. The views expressed herein are those of the author and not necessarily those of the Federal Reserve Bank of Minneapolis or the Federal Reserve System. 


\section{Introduction}

The fact that the children of rich parents have better economic prospects than the children of poor parents ("unequal opportunity") is generally thought to be one of the weak points of modern capitalist societies. The ability of the descendants of poor families to eventually become rich and the descendants of rich families to eventually become poor ("social mobility") is commonly considered to be one of the strong points of these societies. Here I argue that both of these characteristics are, in fact, necessary implications of an efficient societal arrangement when incentives to work hard must be provided.

I argue this point using a generational version of the model of Phelan and Townsend (1991), an infinitely repeated, general equilibrium economy with incentive constraints. In my model, each household's stochastic output is a function of its level of effort. Since effort is assumed to be costly and privately observed (households can shirk), higher than minimal effort levels must be induced by making a household's present or future consumption dependent on the household's observed output history.

A large literature considers models similar to this (including Green (1987), Atkeson and Lucas (1992), Phelan (1994, 1995), Wang (1995), Hopenhayn and Nicolini (1997), and Khan and Ravikumar (2002)). The main difference between the economies in that literature and here is that here, instead of a household consisting of a single infinitely lived individual, a household consists of a sequence of altruistically linked individuals (a familial dynasty), each of whom lives for one period.

This difference affects the appropriate societal ranking of allocations. When households consist of a single infinitely lived individual, allocations can be ranked according to their implied distributions of ex ante lifetime utilities. (If one allocation delivers a distribution of 
ex ante utilities dominated by another allocation, then the first allocation is inefficient.) However, if a household consists of a sequence of altruistically linked individuals, then the appropriate ranking of allocations is no longer obvious. How those in the first generation rank allocations will, in general, differ from how their descendants rank allocations.

This study addresses this conflict between generations by ranking allocations according to a Rawlsian veil-of-ignorance criterion (Rawls (1971)). That is, here, society seeks to maximize the expected dynastic utility (utility including altruism toward descendants) of an individual who does not know into which generation he will be born and does not know the identity or output levels of his ancestors. ${ }^{1}$ I argue that this corresponds to a societal ranking which does not discount the future even though individuals in the society at any given time do discount it. I show this ranking transforms the social planning problem into one of directly choosing the stationary distribution of dynastic utilities, as well as functions for determining effort levels, consumption, and a child's position in this distribution as a function of his parent's output. This transformed problem is a static social choice problem and thus a major simplification.

An immediate result of this societal ranking of allocations (zero discounting) is that efficiency immediately implies a finite amount of inequality in the limit. That is, in previous papers such as Atkeson and Lucas (1992) (which rank allocations according to their ex ante lifetime utilities), efficiency implies inequality grows over time without limit. Limited resources, concave utility, and forever growing inequality imply that mean utility is forever decreasing. Thus while optimal from an ex ante perspective, an allocation with forever grow-

\footnotetext{
${ }^{1}$ Freeman and Sadler (2002) use a similar objective function. They consider whether an optimal policy can be decentralized through inheritances.
} 
ing inequality is not only not efficient, but in fact, the worst one can do if, as in this paper, society does not discount. (Papers subsequent to this paper by Farhi and Werning (2005) and Sleet and Yeltekin (2005) generalize this result. That is, they show a much weaker assumption than zero societal discounting is sufficient to ensure finite limiting inequality. Essentially, they show that as long as the societal ranking puts greater weight on future generations than that implied by the altruism of the first generation, finite limiting inequality will hold.)

My first main theorem is that a society using this ranking will always choose the distribution of dynastic utilities to be nondegenerate; in other words, it will choose unequal opportunity. Some individuals will be born relatively poor (fated to receive relatively low consumption for each output realization), and some will be born relatively rich, even though equal opportunity is feasible. This occurs because it helps with the provision of incentives to make a child poorer if his parent realizes a low output level and richer if his parent realizes a high output level. This, in a sense, extends the result of Rogerson (1985) to a generation context.

My second main theorem, subject to a condition which can be proved for specific functional forms for utility, is that society will choose to have social mobility. It will never choose to have a caste system with one group of families having relatively high average consumption and another having relatively low consumption and with no ability for a family to move between groups. Instead, no matter how poor or rich a parent is, eventually, the expected consumption of that person's descendants equals the unconditional expectation.

The basic intuition behind the first result, unequal opportunity, is that there is zero loss, at the margin, from allowing some dependence of a child's consumption on his parent's output realization. However, a positive marginal benefit results from relaxing the incentive 
constraints on parents by making a child's future consumption depend on his parent's output realization. The basic intuition behind the second result, social mobility, is that a society with multiple castes simply requires more resources to deliver a given mean utility than a single caste society.

After stating and proving these main theoretical results for a general, additively separable utility function, I discuss specific functional forms for utility and computation. Next I show that computation of the optimal stationary distribution of dynastic utilities (along with the functions determining consumption and mobility) consists of solving a single linear program. Finally, I present a computed example and compare it to the static optimum and the optimal allocation when allocations are ranked by ex ante utility, as opposed to limiting, dynastic utility.

At the end, I discuss the generality of these results. I argue that my results do not depend on the particular type of incentive problem discussed here (unobserved effort). My results can apply to unobserved endowment models such as that of Green (1987), unobserved preference shock models such as that of Atkeson and Lucas (1992), and unobserved production models such as that of Khan and Ravikumar (2002).

\section{The Basic Model}

Consider the following economy. In each time period, $t \in\{0, \ldots, \infty\}$, the economy is populated by a unit mass continuum of identical, infinitely lived households. There is a single consumption good. If a household exerts an effort level $a \in A=\left\{a_{0}, \ldots, a_{N}\right\}$, then its output (in terms of the consumption good) $q \in\left\{q_{0}, \ldots, q_{M}\right\}$ occurs with probability $P(q \mid a)$. Assume that $P(q \mid a)>0$ for all $(q, a) \in Q \times A$ and that there exists $(\bar{q}, q) \in Q^{2}$ such that 
if $a_{i}<a_{j}$, then $P\left(\bar{q} \mid a_{i}\right)<P\left(\bar{q} \mid a_{j}\right)$ and $P\left(\underline{q} \mid a_{i}\right)>P\left(\underline{q} \mid a_{j}\right)$. That is, the probability of one outcome (say, the highest) is increasing in $a$, and the probability of another outcome (say, the lowest) is decreasing in $a$. Households are assumed to be able to privately exert effort less than that specified by the allocation. That is, they can shirk.

Household utility in period $t$ is determined by the function $U\left(c_{t}, a_{t}\right)=u\left(c_{t}\right)-v\left(a_{t}\right)$, where $c_{t} \in C$ is the household's period $t$ consumption. (The consumption set $C$ is assumed convex.) The function $u$ is assumed to be unbounded below, twice differentiable with $u^{\prime}>0$ and $u^{\prime \prime}<0$. For $a_{i}<a_{j}, v\left(a_{i}\right)$ is assumed to be strictly less than $v\left(a_{j}\right)$. Over time and uncertainty, a household cares about the expected value of $(1-\beta) \sum_{t=0}^{\infty} \beta^{t} U\left(c_{t}, a_{t}\right)$, where $\beta \in(0,1)$. Let $V=\left\{u(c)-v\left(a_{0}\right) \mid c \in C\right\}$.

Define the efficient symmetric static allocation $\left(a^{*}, c^{*}(q)\right)$ as the solution to

$$
\max _{a, c(q)} \sum_{q} P(q \mid a) u(c(q))-v(a)
$$

subject to a static resource constraint

$$
\sum_{q} P(q \mid a)(c(q)-q) \leq 0
$$

and the static incentive constraint (for $\hat{a}<a$ )

$$
\sum_{q} P(q \mid a) u(c(q))-v(a) \geq \sum_{q} P(q \mid \hat{a}) u(c(q))-v(\hat{a}) .
$$




\section{A. Dynamic Allocations}

Let a dynamic allocation (or simply an allocation) ( $\Psi_{0},\left\{a_{t}\left(w_{t}\right), c_{t}\left(w_{t}, q_{t}\right)\right.$, $\left.\left.w_{t+1}\left(w_{t}, q_{t}\right)\right\}_{t=0}^{\infty}\right)$ be defined recursively as a measure of initial lifetime utilities, $\Psi_{0}$, and a sequence of functions $a_{t}\left(w_{t}\right), c_{t}\left(w_{t}, q_{t}\right)$, and $w_{t+1}\left(w_{t}, q_{t}\right)$. The function $a_{t}\left(w_{t}\right)$ specifies the recommended effort level for a household which starts period $t$ with a continuation expected utility of $w_{t}$. The function $c_{t}\left(w_{t}, q_{t}\right)$ specifies the nonnegative consumption of a household which starts period $t$ with a continuation expected utility of $w_{t}$ and realizes output $q_{t}$. The function $w_{t+1}\left(w_{t}, q_{t}\right)$ specifies the continuation expected discounted utility at the beginning of period $t+1$ of a household which starts period $t$ with a continuation expected utility of $w_{t}$ and realizes output $q_{t}$

Note that through the initial distribution of forward-looking utilities $\Psi_{0}$, and the functions $a_{t}\left(w_{t}\right)$ and $w_{t+1}\left(w_{t}, q_{t}\right)$, an allocation determines, for all $t \geq 1$, the period $t$ distribution of forward-looking utilities $\Psi_{t}$.

An allocation is said to satisfy promise-keeping if, for all $t$ and $w_{t}$,

$$
w_{t}=\sum_{q_{t}} P\left(q_{t} \mid a_{t}\left(w_{t}\right)\right)\left((1-\beta)\left[u\left(c_{t}\left(w_{t}, q_{t}\right)\right)-v\left(a_{t}\left(w_{t}\right)\right)\right]+\beta w_{t+1}\left(w_{t}, q_{t}\right)\right) .
$$

In words, promise-keeping requires that expected dynastic utility of an allocation conditional on $w_{t}$ is actually $w_{t}$. Next, an allocation is said to be incentive-compatible if, for all $t, w_{t}$, and $\hat{a}<a_{t}\left(w_{t}\right)$,

(2) $\quad w_{t} \geq \sum_{q_{t}} P\left(q_{t} \mid \hat{a}\right)\left((1-\beta)\left[u\left(c_{t}\left(w_{t}, q_{t}\right)\right)-v(\hat{a})\right]+\beta w_{t+1}\left(w_{t}, q_{t}\right)\right)$. 
Here, the left side is the dynastic utility associated with taking action $a_{t}\left(w_{t}\right)$, and the right side is the dynastic utility associated with taking an alternative action $\hat{a}<a_{t}\left(w_{t}\right)$. Finally, an allocation is said to be resource-feasible if, for all $t$,

$$
0 \geq \int_{V} \sum_{q} P\left(q \mid a_{t}\left(w_{t}\right)\right)\left[c\left(w_{t}, q\right)-q\right] d \Psi_{t}\left(w_{t}\right) .
$$

Condition (3) requires that aggregate production be weakly greater than aggregate consumption. A dynamic allocation is considered feasible if it satisfies all three of these conditions $(1)-(3) .^{2}$

\section{B. Ranking Feasible Allocations}

In most dynamic contracting work, a household consists of a single infinitely lived individual who discounts the future by $\beta .^{3}$ Given this, an allocation is considered efficient if it is feasible (satisfies promise-keeping, incentive-compatibility, and resource-feasibility) and if no other feasible allocation delivers a distribution of initial utilities which dominates $\Psi_{0}$.

In models similar to this one, Atkeson and Lucas (1992) and Phelan (1994) derive several implications of this type of efficiency. First, these studies show that an efficient allocation must, household by household, minimize the discounted resource cost of delivering a given ex ante utility $w_{0}$ and that this resource cost is a convex function of $w_{0}$. Thus, a society maximizing mean ex ante utility would choose a degenerate measure of initial utilities

\footnotetext{
${ }^{2}$ The recursive formulation also requires a no-Ponzi scheme condition to ensure that arbitrarily high dynastic utilities are not delivered by promising ever higher future dynastic utilities. One sufficient condition is that for all $t$, mean dynastic utility be weakly less than $u\left(q_{M}\right)-v\left(a_{0}\right)$, the utility of every day consuming the highest output level and taking the lowest effort.

${ }^{3}$ See Green (1987), Phelan and Townsend (1991), and Atkeson and Lucas (1992) among many others. An exception is Freeman and Sadler (2002).
} 
$\Psi_{0}$ with all mass on the same point. ${ }^{4}$ Second, these studies show that efficiency, by this definition, implies extreme results regarding the limiting distribution of consumption and utility. In the model of Atkeson and Lucas (1992), almost all consumption paths go to zero, and mean utility goes to the lower bound of the set of possible utilities (either zero or negative infinity, depending on the level of risk aversion). In the model of Phelan (1994), the variance of consumption grows without bound, and thus mean utility becomes infinitely negative.

Here I consider a different ranking of allocations, supported by the following assumption. Instead of a household consisting of a single infinitely lived individual, suppose that a household consists of a sequence of altruistically linked individuals, each of whom lives for one period. Specifically, assume that the dynastic utility of an individual born in period $t$ consists of weight $1-\beta$ on his own direct utility $U\left(c_{t}, a_{t}\right)$ and weight $\beta$ on the dynastic utility of his single child. Thus, his dynastic utility is

$$
w_{t}=\sum_{q_{t}} P\left(q_{t} \mid a_{t}\left(w_{t}\right)\right)\left[(1-\beta) U\left(c_{t}\left(w_{t}, q_{t}\right), a_{t}\left(w_{t}\right)\right)+\beta w_{t+1}\left(w_{t}, q_{t}\right)\right]
$$

(This is equivalent to the individual putting weight $1-\beta$ on his own direct utility, weight $\beta(1-\beta)$ on his child's direct utility, $\beta^{2}(1-\beta)$ on his grandchild's, and so on.) With this composition of households, the set of feasible allocations is identical to that which holds if households consist of a single infinitely lived individual. However, the appropriate ranking of allocations is no longer obvious. Maximizing ex ante dynastic utility puts no direct weight on the utility of generations born later than period $t=0$. These later generations enter the

\footnotetext{
${ }^{4}$ Ranking allocations by mean ex ante utility is equivalent to maximizing the utility of a household which does not know where in distribution $\Psi_{0}$ it will be, but instead sees itself as having the same probability as all other households of being in any subset of the support of $\Psi_{0}$.
} 
social calculus only indirectly, through the altruism of those born in period $t=0$. Pareto efficiency, rather than putting all weight on the first generation, would allow any arbitrary weighting scheme across generations.

This paper considers an alternative extreme of equal weighting of all generations, or, put differently, zero societal discounting. Formally, let $\bar{v}_{t}=\int_{V} w_{t} d \Psi_{t}\left(w_{t}\right)$ and $\bar{v}=$ $\liminf \operatorname{in}_{T \rightarrow \infty} \frac{1}{T+1} \sum_{t=0}^{T} \bar{v}_{t}{ }^{5}$ In words, $\bar{v}_{t}$ is the average utility, including altruism toward children, of individuals born in period $t$ and $\bar{v}$ is the limiting average of this average over dates. Instead of ranking allocations by $v_{0}$, allocations can be ranked by $\bar{v}$. Since the number of periods is infinite, such a weighting scheme puts zero weight on the first $T$ periods (regardless of $T$ ). (Of course, one can admit intermediate weighting schemes where later generations have positive direct weight, but later generations have successively less weight. That is, one could rank allocations by $\sum_{t=0}^{\infty} \delta^{t} \bar{v}_{t}$ where $\delta<1$. Two recent papers by Farhi and Werning (2005) and Sleet and Yeltekin (2005) build on this work and consider precisely this intermediate case. )

It is useful to establish that a plan which maximizes limiting mean utility, $\bar{v}$, minimizes limiting mean resources. Attention will then be mostly restricted to cost minimizing, rather than mean limiting utility maximizing allocations. To this end, for $\zeta=\left(\Psi_{0},\left\{a_{t}\left(w_{t}\right), c_{t}\left(w_{t}, q_{t}\right)\right.\right.$, $\left.\left.w_{t+1}\left(w_{t}, q_{t}\right)\right\}_{t=0}^{\infty}\right)$, let

$$
S_{t}(\zeta)=\int_{V} \sum_{q} P\left(q \mid a_{t}\left(w_{t}\right)\right)\left[c\left(w_{t}, q\right)-q\right] d \Psi_{t}\left(w_{t}\right)
$$

\footnotetext{
${ }^{5}$ An earlier version ranks allocations by $\lim _{T \rightarrow \infty} \frac{1}{T+1} \sum_{t=0}^{T} \bar{v}_{t}$, but this limit may not exist. A standard approach in game theory to implementing zero discounting is ranking by the lim inf. (See Fudenberg and Tirole (1991), p. 148.) The author thanks an anonymous referee for pointing out the error.
} 
and

$$
S(\zeta)=\limsup _{T \rightarrow \infty} \frac{1}{T+1} \sum_{t=0}^{T} S_{t}(\zeta)
$$

Call the primal problem $\max _{\zeta} \liminf _{T \rightarrow \infty} \frac{1}{T+1} \sum_{t=0}^{T} v_{t}$ subject to (1), (2), and (3). Call the dual problem $\min _{\zeta} S(\zeta)$ subject to $\bar{v} \leq \liminf _{T \rightarrow \infty} \frac{1}{T+1} \sum_{t=0}^{T} \int_{V} w d \Psi_{t}(w)$ and constraints (1) and (2).

LEMma 1. If allocation $\zeta$ solves the primal problem with objective function value $\bar{v}$, it solves the dual problem with $S(\zeta)=0$ when limiting average utility must equal $\bar{v}$.

(Proof relegated to Appendix.)

\section{Stationary Allocations}

Let a stationary allocation be defined as a dynamic allocation where for all $t$ and $s$, $\left(\Psi_{t}, a_{t}(w), c_{t}(w, q), w_{t+1}^{\prime}(w, q)\right)=\left(\Psi_{s}, a_{s}(w), c_{s}(w, q), w_{s+1}^{\prime}(w, q)\right)$. Alternatively, a stationary allocation is a collection $\xi=\left(\Psi, a(w), c(w, q), w^{\prime}(w, q)\right)$ such that for all subsets $W \in \mathcal{B}(V)$,

$$
\Psi(W)=\int_{V} \sum_{q} P(q \mid a(w)) I\left(w^{\prime}(w, q) \in W\right) d \Psi(w),
$$

where $I(\cdot)$ is the indicator function and $\mathcal{B}(V)$ denotes the Borel subsets of $V$. Here, the left side is the mass of households on set $W$ today, and the right side is the mass of households on set $W$ tomorrow. 
A stationary allocation satisfies promise-keeping if for almost all $w$ relative to $\Psi$,

$$
w=\sum_{q} P(q \mid a(w))\left((1-\beta)[u(c(w, q))-v(a(w))]+\beta w^{\prime}(w, q)\right)
$$

In words, condition (5) requires that the functions $a(w), c(w, q)$, and $w^{\prime}(w, q)$ actually deliver dynastic utility $w$.

A stationary allocation is considered incentive-compatible if for almost all $w$ relative to $\Psi$ and all $\hat{a}<a(w)$,

$$
w \geq \sum_{q} P(q \mid \hat{a})\left((1-\beta)[u(c(w, q))-v(\hat{a})]+\beta w^{\prime}(w, q)\right)
$$

As before, the left side is the dynastic utility of taking action $a(w)$, and the right side is the dynastic utility of taking alternative action $\hat{a}$.

Finally, a stationary allocation satisfies resource-feasibility if

$$
0 \geq \int_{V} \sum_{q} P(q \mid a(w))[c(w, q)-q] d \Psi(w)
$$

Call the stationary primal problem $\max _{\xi} \int_{V} w d \Psi(w)$ subject to (4)-(7). Call the stationary dual problem $\min _{\xi} \int_{V} \sum_{q} P(q \mid a(w))[c(w, q)-q] d \Psi(w)$ subject to (4)-(6) and the mean utility constraint $\bar{v} \leq \int_{V} w d \Psi(w)$.

The next lemma shows that without loss, attention can be restricted to stationary allocations. This is a major simplification since the stationary primal and dual problems are static optimizations. The main idea behind the proof is that any dynamic allocation for which $\Psi_{t}$ does not converge can be used to create another dynamic allocation where $\Psi_{t}$ does 
converge where the difference in cost between the two allocations is arbitrarily small. Once attention can be restricted to allocations where $\Psi_{t}$ converges, given zero discounting ranking of allocations, restricting attention to stationary allocations is immediate.

Lemma 2. Suppose $\xi=\left(\Psi, a(w), c(w, q) \cdot w^{\prime}(w, q)\right)$ solves the stationary dual problem. Then $\left(\Psi_{0}=\Psi,\left\{a_{t}\left(w_{t}\right)=a\left(w_{t}\right), c_{t}\left(w_{t}, q_{t}\right)=c\left(w_{t}, q_{t}\right), w_{t}^{\prime}\left(w_{t}, q_{t}\right)=w^{\prime}\left(w_{t}, q_{t}\right)\right\}_{t=0}^{\infty}\right)$ solves the dual problem.

(Proof relegated to Appendix.)

\section{Characterizing Optimal Allocations}

This section presents the main results of the paper: unequal opportunity and social mobility are necessarily characteristics of an optimal allocation. Showing this requires several supporting lemmas (with again the proofs relegated to the appendix).

LEMma 3. If a stationary allocation $\xi^{*}=\left(\Psi^{*}, a^{*}(w), c^{*}(w, q), w^{\prime *}(w, q)\right)$ solves the stationary primal problem, then $\int_{V} \sum_{q} P\left(q \mid a^{*}(w)\right)\left[c^{*}(w, q)-q\right] d \Psi^{*}(w)=0$. Likewise, if stationary allocation $\xi^{*}=\left(\Psi^{*}, a^{*}(w), c^{*}(w, q), w^{\prime *}(w, q)\right)$ solves the stationary dual problem, then the mean utility constraint $\bar{v} \leq \int_{V} w d \Psi^{*}(w)$ holds as an equality.

Given that the resource constraint (7) binds in the stationary primal problem and the mean utility constraint binds in the stationary dual problem, it is straightforward to show that a plan which maximizes mean utility minimizes the cost of providing any given mean utility, and vice versa. Thus, the second supporting lemma is the following.

LEMma 4. Suppose a stationary allocation $\xi^{*}$ solves the stationary primal problem, and let 
$v=\int_{V} w d \Psi^{*}(w)$. Then $\xi^{*}$ solves the stationary dual problem

$$
C(\bar{v})=\min _{\Psi, a(w), c(w, q), w^{\prime}(w, q)} \int_{V} \sum_{q} P(q \mid a(w))[c(w, q)-q] d \Psi(w)
$$

subject to (4)-(6), and

(9) $\quad v \leq \int_{V} w d \Psi(w)$

with $C(\bar{v})=0$. Likewise, if $\xi^{*}$ solves the stationary dual problem with $C(\bar{v})=0$, it solves the stationary primal problem.

\section{A. Opportunity}

Lemma 4 allows for the first main result, that an optimal plan will always exhibit unequal opportunity. (Some individuals are born with lower expected dynastic utility than others.) The general strategy of the proof is to assume that all incentives are static - that all individuals are born with a blank slate - and show that the cost of introducing a small amount of dependency of children's consumption on parents' outcomes is second-order, while the benefit, or gain, from this dependency (which allows for the better provision of incentives to parents) is first-order.

Theorem 1. Suppose $\xi^{*}=\left(\Psi^{*}, a^{*}(w), c^{*}(w, q), w^{\prime *}(w, q)\right)$ solves the stationary dual problem. Then $\Psi^{*}$ is nondegenerate.

Proof. The strategy of this proof is similar to that in Rogerson (1985): Suppose no links across periods and show there exists an improving perturbation. (The model of this 
paper is sufficiently different from the model in Rogerson (1985) that this proof needs a different perturbation than that in Rogerson (1985).) To this end, suppose $\Psi^{*}$ is degenerate with all mass on point $w^{*}$. Let $a^{*}=a^{*}\left(w^{*}\right)$. Define an alternative allocation $\xi=\left(\Psi, a(w), c(w, q), w^{\prime}(w, q)\right)$. First, let $\Psi$ put mass $1-P\left(\underline{q} \mid a^{*}\right)-P\left(\bar{q} \mid a^{*}\right)$ on point $w^{*}$, mass $P\left(\underline{q} \mid a^{*}\right)$ on point $\underline{w}=w^{*}-\epsilon / P\left(\underline{q} \mid a^{*}\right)$, and mass $P\left(\bar{q} \mid a^{*}\right)$ on point $\bar{w}=w^{*}+\epsilon / P\left(\bar{q} \mid a^{*}\right)$. By construction, then $\int_{V} w d \Psi(w)=\int_{V} w d \Psi^{*}(w)=w^{*}$; thus, $\xi$ satisfies condition (9) for $\bar{v}=w^{*}$. Next, assume for $w \in\left\{\underline{w}, w^{*}, \bar{w}\right\}$ that $a(w)=a^{*}$. This ensures that aggregate production is unchanged.

Next, let $w^{\prime}(w, \underline{q})=\underline{w}, w^{\prime}(w, \bar{q})=\bar{w}$, and for $q \notin\{\underline{q}, \bar{q}\}, w^{\prime}(w, q)=w^{*}$. This ensures (for all $\epsilon$ ) that stationarity (4) is satisfied. Lastly, define the functions $c(w, q)$ for $(w, q) \in\left\{\underline{w}, w^{*}, \bar{w}\right\} \times Q$. To do this, for all $q$, let $c\left(w^{*}, q\right)$ be such that $u\left(c\left(w^{*}, q\right)\right)=$ $u\left(c^{*}\left(w^{*}, q\right)\right)+\Delta\left(w^{*}, q\right)$. Next, let $c(\underline{w}, q)$ be such that $u(c(\underline{w}, q))=u\left(c^{*}\left(w^{*}, q\right)\right)-\epsilon /[(1-$ $\left.\beta) P\left(\underline{q} \mid a^{*}\right)\right]+\Delta(\underline{w}, q)$. Finally, let $c(\bar{w}, q)$ be such that $u(c(\bar{w}, q))=u\left(c^{*}\left(w^{*}, q\right)\right)+\epsilon /[(1-$ $\left.\beta) P\left(\bar{q} \mid a^{*}\right)\right]+\Delta(\bar{w}, q)$. Since the original stationary allocation $\xi^{*}$ is optimal, choosing $\epsilon=0$ and $\Delta(w, q)=0$ for all $(w, q) \in\left\{\underline{w}, w^{*}, \bar{w}\right\} \times Q$ must minimize equation (8) subject to the promise-keeping constraint (5) and the incentive-compatibility constraint (6).

Note that the incentive constraint associated with $w=w^{*}$ in this restricted optimization problem binds; thus, the marginal value of loosening it is strictly positive. To see this, consider choosing $\epsilon$ and $\Delta(w, q)$ to minimize (8) subject to the promise-keeping constraint (5) but not the incentive constraint (6). Here, I can strictly improve on $\xi^{*}$ by setting $\epsilon=0$ and setting $\Delta\left(w^{*}, q\right)$ such that $c\left(w^{*}, q\right)=\sum_{q} P\left(q \mid a^{*}\right) c^{*}\left(w^{*}, q\right)$ (full consumption insurance) less a constant to compensate for the utility gain associated with full insurance. (Recall the assumption that $a^{*}>a_{0}$. Thus, full consumption insurance is not attained by $\xi^{*}$.) 
Now set $\Delta(w, q)=0$ for all $(w, q)$. This ensures that the promise-keeping constraint (5) holds for all $\epsilon$. Thus, a choice of $\epsilon \neq 0$ affects only the incentive constraint (6) and the dual objective function (8).

For $w \in\left\{\underline{w}, w^{*}, \bar{w}\right\}$, the derivative, with respect to $\epsilon$, of the left side of the incentive constraint minus the derivative of the right side equals $\beta\left[P(\bar{q} \mid \hat{a}) / P\left(\bar{q} \mid a^{*}\right)-P(\underline{q} \mid \hat{a}) / P\left(\underline{q} \mid a^{*}\right)\right]$. This derivative is a strictly negative constant (not a function of $\epsilon$ ) for all $\hat{a}<a^{*}$, and thus, the incentive constraint for each $w$ is loosened as $\epsilon$ increases.

Finally, let $u^{-1}(u)$ denote the consumption payment necessary to deliver utility $u(c)$. The dual objective function with the definition of $\xi$ and $\Delta(w, q)=0$ substituted in is then

$$
\begin{array}{r}
P\left(\underline{q} \mid a^{*}\right) \sum_{q} P\left(q \mid a^{*}\right)\left[u^{-1}\left(u\left(c^{*}\left(w^{*}, q\right)\right)-\frac{\epsilon}{P\left(\underline{q} \mid a^{*}\right)}\right)-q\right] \\
+P\left(\bar{q} \mid a^{*}\right) \sum_{q} P\left(q \mid a^{*}\right)\left[u^{-1}\left(u\left(c^{*}\left(w^{*}, q\right)\right)+\frac{\epsilon}{P\left(\bar{q} \mid a^{*}\right)}\right)-q\right] \\
+\left[1-P\left(\underline{q} \mid a^{*}\right)-P\left(\bar{q} \mid a^{*}\right)\right] \sum_{q} P\left(q \mid a^{*}\right)\left[u^{-1}\left(u\left(c^{*}\left(w^{*}, q\right)\right)\right)-q\right] .
\end{array}
$$

The derivative of this expression with respect to $\epsilon$ is

$$
\begin{aligned}
& -\sum_{q} P\left(q \mid a^{*}\right) u^{-1^{\prime}}\left(u\left(c^{*}\left(w^{*}, q\right)\right)-\frac{\epsilon}{P\left(\underline{q} \mid a^{*}\right)}\right) \\
& +\sum_{q} P\left(q \mid a^{*}\right) u^{-1^{\prime}}\left(u\left(c^{*}\left(w^{*}, q\right)\right)+\frac{\epsilon}{P\left(\bar{q} \mid a^{*}\right)}\right) .
\end{aligned}
$$

This derivative equals zero for $\epsilon=0$. Thus, the marginal value of increasing $\epsilon$ when $\epsilon=0$ and $\Delta(w, q)=0$ is strictly positive since it loosens a binding constraint (a first-order benefit) with zero first-order effect on the objective function, contradicting the optimality of the original allocation. 


\section{B. Social Mobility}

Note that an allocation $\xi=\left(\Psi, a(w), c(w, q), w^{\prime}(w, q)\right)$ defines not only the distribution of dynastic utilities, $\Psi$, but also the rules under which households move up or down this distribution. Thus, the answers to questions regarding social mobility are embedded in $\xi$. (Can the descendants of poor, or low w, households eventually become rich?) Now I consider to what extent the efficiency of $\xi$ implies social mobility. In particular, I argue that social mobility is a direct implication of strict convexity of the cost function $C(\bar{v})$.

To allow a strict definition of social mobility, let a set $W \in \mathcal{B}(V)$ be called a caste under stationary allocation $\xi$ if $\Psi(W)>0$, and

$$
\Psi(W)=\int_{W} \sum_{q} P(q \mid a(w)) I\left(w^{\prime}(w, q) \in W\right) d \Psi(w)
$$

which implies a zero exit and entry probability from $W$. A caste $W$ is called trivial (relative to $\xi)$ if $\Psi(W)=1$, or if $\int_{W} w d \Psi(w)=\int_{W^{c}} w d \Psi(w)$, where $W^{c}$ denotes the complement of $W$. Thus, for a caste system to be nontrivial, its complement must have positive mass and the mean utility of those in the caste must differ from the mean utility of those outside the caste.

My main theorem here is that if $C(\bar{v})$ is strictly convex (a condition shown in the next section for particular functional forms), then any caste system must be trivial. That is, having a permanently richer group and a permanently poorer group is never optimal.

THEOREM 2. Suppose $C(\bar{v})$ is strictly convex and a stationary allocation $\xi=(\Psi, a(w), c(w, q)$, $\left.w^{\prime}(w, q)\right)$ minimizes (8) subject to (4)-(6), and (9) for $\bar{v}=\int w \Psi w(w)$. Then any caste $W$ relative to $\xi$ is trivial. 
Proof. Let $W_{1}$ be a caste relative to $\xi$. If $\Psi\left(W_{1}\right)=1$, then the result is proved; thus, assume that $\Psi\left(W_{1}\right)<1$. Let $W_{2}=W_{1}^{c}$. Like $W_{1}$, the set $W_{2}$ is also a caste. Define two separate allocations $\xi_{i}, i \in\{1,2\}$, by choosing $\Psi_{i}$ such that for all $W \subset W_{i}, \Psi_{i}(W)=\Psi(W) / \Psi\left(W_{i}\right)$ and leaving the functions $a(w), c(w, q)$, and $w^{\prime}(w, q)$ unaltered. (That is, proportionally put all mass on one set or the other, but otherwise change nothing). These allocations each satisfy promise-keeping and incentive-compatibility since the original allocation satisfies these conditions, and each satisfies stationarity since $W_{1}$ and $W_{2}$ do not communicate and the original allocation satisfied stationarity. Put less formally, the fact that the sets $W_{1}$ and $W_{2}$ don't communicate implies that how those in each set are treated defines a feasible allocation for treating all of society. Thus, each allocation must minimize (8) subject to (4)-(6), and (9) for $\bar{v}=w_{i}$, where $w_{i}=\left[1 / \Psi\left(W_{i}\right)\right] \int_{V} w d \Psi_{i}(w), i \in\{1,2\}$. If another allocation satisfies (4)-(6) and (9) at a lower cost, then the original allocation $\xi$ could not have been optimal, since this lower cost allocation could have been incorporated into the original allocation, lowering its cost. Finally, if $w_{1} \neq w_{2}$, then the strict convexity of $C$ implies that $C\left(\Psi\left(W_{1}\right) w_{1}+\Psi\left(W_{2}\right) w_{2}\right)<\Psi\left(W_{1}\right) C\left(w_{1}\right)+\Psi\left(W_{2}\right) C\left(w_{2}\right)$. Since the right side of this inequality is the resource cost of the original plan, $w_{1}=w_{2}$.

\section{Functional Forms}

Here I introduce two explicit functional forms for $U(c, a)$. These functional forms allow me to solve for $C(\bar{v})$ (up to a constant) and thus prove the strict convexity assumed by Theorem 2. Further, they allow for a relatively complete characterization of the optimal allocation when allocations are ranked by ex ante utility, as opposed to limiting, mean utility; they thus help highlight the effect of ranking allocations by the mean of the limiting distribution of 
dynastic utilities. While these examples are not additively separable (as the earlier sections assumed), Lemmas 1 and 2 and Theorem 1 can be proved using arguments similar to those used earlier.

The simplest example has $U(c, a)=-\exp (-\gamma[c-v(a)])$ with consumption unbounded (or $c \in \mathbb{R}$ ) and $\gamma>0$ - the constant absolute risk aversion (CARA) utility specification in Phelan (1994). Given this utility function and consumption set, Phelan (1994) shows that if allocations are ranked by mean ex ante utility, optimality implies that $a_{t}$ is constant across households and time (thus, so is aggregate production), and household consumption is the sum of an independent and identically distributed random variable and a term which follows a driftless random walk. Since effort is constant and utility is a concave function of consumption, as the cross-sectional variance of consumption increases due to the random walk term, mean dynastic utility decreases over time without bound. In essence, the optimal allocation from an ex ante perspective implies a limiting distribution of dynastic utilities which has all mass on negative infinity. However, that's the worst possible allocation when allocations are ranked, as they are here, by the mean of the limiting distribution of dynastic utilities. (Theorem 1 shows that a finite mean limiting utility can, in fact, be achieved, since one can do better than repeating the static optimum, which itself has a finite mean utility.)

Assuming this specific functional form also allows me to show that $C(\bar{v})$ is strictly convex, as assumed by Theorem 2. This is shown in the following lemma:

Lemma 5. If $U(c, a)=-\exp (-\gamma[c-v(a)])$ with $c \in \mathbb{R}$ and $\gamma>0$, then for $v<0$,

$$
C(\bar{v})=\min _{\Psi, a(w), c(w, q), w^{\prime}(w, q)} \int_{V} \sum_{q} P(q \mid a(w))[c(w, q)-q] d \Psi(w)
$$


subject to (4)-(6), and (9) satisfies $C(\bar{v})=-\log (-\bar{v}) / \gamma+C(-1)$ and is thus strictly convex.

Proof. See the Appendix.

With some extension of the model, I can construct tractable example economies which do not depend on CARA utility. In particular, following Atkeson and Lucas (1992), Khan and Ravikumar (2002), and Phelan (2002), let household output equal $k q$, where $k$ is the quantity of land allocated to the household for use in production that period. Let $v(a)$ denote the per-acre utility loss to effort, and thus, let $k v(a)$ denote the total utility loss to effort $a$. Finally, let utility be the constant relative risk aversion (CRRA) specification $U(c, a, k)=[c-k v(a)]^{\gamma} / \gamma$, where $\gamma=0$ implies that $U(c, a, k)=\log [c-k v(a)]$. With this specification, if allocations are ranked by ex ante utility, almost all household consumption paths converge to zero, the result of Atkeson and Lucas (1992) for a preference shock model. This implies that the limiting distribution of dynastic utilities has either all mass on zero (for the case of $\gamma>0$ ) or all mass on negative infinity (for the case of $\gamma \leq 0$ ). Here, as in the previous example, if allocations are ranked by the mean of the limiting distribution, analogs of Lemmas 1 and 2 and Theorem 1 can be proved.

Introducing land to the model introduces an additional resource constraint into the primal problem. Not only must society not allocate more of the consumption good than is available from production, it must also not allocate more land to households than is exogenously given. However, if society is assumed to be able to trade land for the consumption good at a linear price $p$ (which can be set equal to the ratio of the Lagrange multipliers associated with the separate resource constraints), then I can prove that $C(\bar{v})$ is convex. This is shown in the following lemma. 
LEMMA 6. If $U(c, a, k)=[c-k v(a)]^{\gamma} / \gamma$ with $c \geq k v(a)$, then

$$
C(\bar{v})=\min _{\Psi, k(w), a(w), c(w, q), w^{\prime}(w, q)} \int_{V}\left(p k(w)+\sum_{q} P(q \mid a(w))[c(w, q)-q]\right) d \Psi(w)
$$

subject to (4)-(6), and (9) satisfies $C(\bar{v})=\bar{v}^{1 / \gamma} C(1)$ if $\gamma>0$ (and thus $\bar{v}>0$ ), $C(\bar{v})=$ $\exp (\bar{v}) C(0)$ if $\gamma=0$, and $C(\bar{v})=(-\bar{v})^{1 / \gamma} C(-1)$ if $\gamma<0$ (and, thus, $\bar{v}<0$ ). In each case, $C(\bar{v})$ is strictly convex.

Proof. See the Appendix.

\section{Computation}

My approach of directly choosing the stationary allocation simplifies computation as well. In particular, if $\Psi$ is restricted to a finite support, then an optimal stationary allocation can be computed as a single linear program along the lines of Prescott and Townsend (1984). While the incentive constraints capture the dynamic decision of households, the choice of how to organize society given those constraints is static; thus, dynamic programming along the lines of Phelan and Townsend (1991) or Atkeson and Lucas (1992) is not needed.

The linear program is set up as follows. Let $\hat{V} \subset V$ be a finite grid of points in $V$

restricting the support of $\Psi$. Next, let $\hat{C}$ be a finite grid of points restricting the range of $c(w, q)$. (The function $a(w)$ has already been assumed to have a finite range.) The key to transforming the choice of the stationary allocation into a linear program is to combine the measure $\Psi$ (now restricted to a finite support) with the rules $a(w), c(w, q)$, and $w^{\prime}(w, q)$. That is, let $\mu\left(w, a, q, c, w^{\prime}\right)$ be the fraction of households who start at point $w \in \hat{V}$, receive action recommendation $a \in A$, experience output realization $q \in Q$, get consumption level 
$c \in \hat{C}$, and transit to point $w^{\prime} \subset \hat{V}$.

Choosing $\mu\left(w, a, q, c, w^{\prime}\right)$ for all $\left(w, a, q, c, w^{\prime}\right) \in \hat{V} \times A \times Q \times \hat{C} \times \hat{V}$ pins down $\left(\Psi, a(w), c(w, q), w^{\prime}(w, q)\right)$ if $\mu\left(w, a, q, c, w^{\prime}\right)$ satisfies several linear conditions. First, the fractions $\mu\left(w, a, q, c, w^{\prime}\right)$ must add to one, or

$$
\sum_{w, a, q, c, w} \mu\left(w, a, q, c, w^{\prime}\right)=1
$$

Next, the fraction of households which realize output $q$ must coincide with the fraction determined by the technology $P(q \mid a)$. This can be enforced by requiring that Bayes' rule holds for all $(\bar{w}, \bar{a}, \bar{q}) \in \hat{V} \times A \times Q$ :

$$
\sum_{c, w^{\prime}} \mu\left(\bar{w}, \bar{a}, \bar{q}, c, w^{\prime}\right)=\pi(\bar{q} \mid \bar{a}) \sum_{q, c, w^{\prime}} \mu\left(\bar{w}, \bar{a}, q, c, w^{\prime}\right)
$$

The objective function, stationarity, the promise-keeping, incentive-compatibility, and resource-feasibility constraints are, like the previous conditions, linear in the choice variables. The primal objective function becomes

$$
\sum_{w, a, q, c, w^{\prime}} \mu\left(w, a, q, c, w^{\prime}\right) w
$$

A collection $\mu\left(w, a, q, c, w^{\prime}\right)$ is stationary if for all $w \in \hat{V}$, the fraction of households at $w$ is the same today and tomorrow, or if for all $\bar{w} \in \hat{V}$,

$$
\sum_{a, q, c, w^{\prime}} \mu\left(\bar{w}, a, q, c, w^{\prime}\right)=\sum_{w, a, q, c} \mu(w, a, q, c, \bar{w}) .
$$


Promise-keeping requires that, for all $w \in \hat{V}$,

$$
\sum_{a, q, c, w^{\prime}} \mu\left(w, a, q, c, w^{\prime}\right)\left[(1-\beta) u(c, a)+\beta w^{\prime}-w\right]=0
$$

Incentive-compatibility requires that, for all $(w, a, \hat{a}<a)$,

$$
\begin{aligned}
& \sum_{q, c, w^{\prime}} \mu\left(w, a, q, c, w^{\prime}\right)\left[(1-\beta) u(c, a)+\beta w^{\prime}\right] \geq \\
& \sum_{q, c, w^{\prime}} \mu\left(w, a, q, c, w^{\prime}\right)\left[(1-\beta) u(c, \hat{a})+\beta w^{\prime}\right] \frac{P(q \mid \hat{a})}{P(q \mid a)}
\end{aligned}
$$

Finally, the resource-feasibility constraint is satisfied if and only if

$$
\sum_{w, a, q, c, w^{\prime}} \mu\left(w, a, q, c, w^{\prime}\right)(c-q) \leq 0
$$

\section{An Example}

Now I present an example economy computed using the methods just outlined. For this example economy, I also compute the solutions to the static optimum and the optimum when allocations are ranked by ex ante dynastic utility and compare those solutions to that when allocations are ranked by the mean of $\Psi$, the stationary distribution of dynastic utilities.

In the example, these are the parameter values used: $a \in\{0,1\}, q \in\{0,1\}, \beta=2 / 3$, and $U(c, a)=-\exp (-(c-0.3 a))$. The high output $(q=1)$ occurs with probability $3 / 4$ if $a=1$ and probability $1 / 4$ if $a=0 .^{6}$

\footnotetext{
${ }^{6}$ The parameters specific to the computation method are as follows: $\hat{V}=\{-0.90,-0.88, \ldots,-0.52,-0.50\}$ and $\hat{C}=\{-0.20,-0.18, \ldots, 1.02,1.04\}$. The program was written in $\mathrm{C}$ using the gnu compiler and the gnu linear programming package. While the resulting linear program has 111,132 variables and 106 constraints, it solves on an Apple 867MHz PowerBook G4 in under four minutes.
} 
Figure 1 displays $\Psi$, as well as the utility levels associated with the static optimum, the mean of $\Psi$, and the dynastic utility of the first generation when allocations are ranked by their ex ante dynastic utility. (The ex-ante optimal allocation has a degenerate limiting distribution with all mass at negative infinity.)

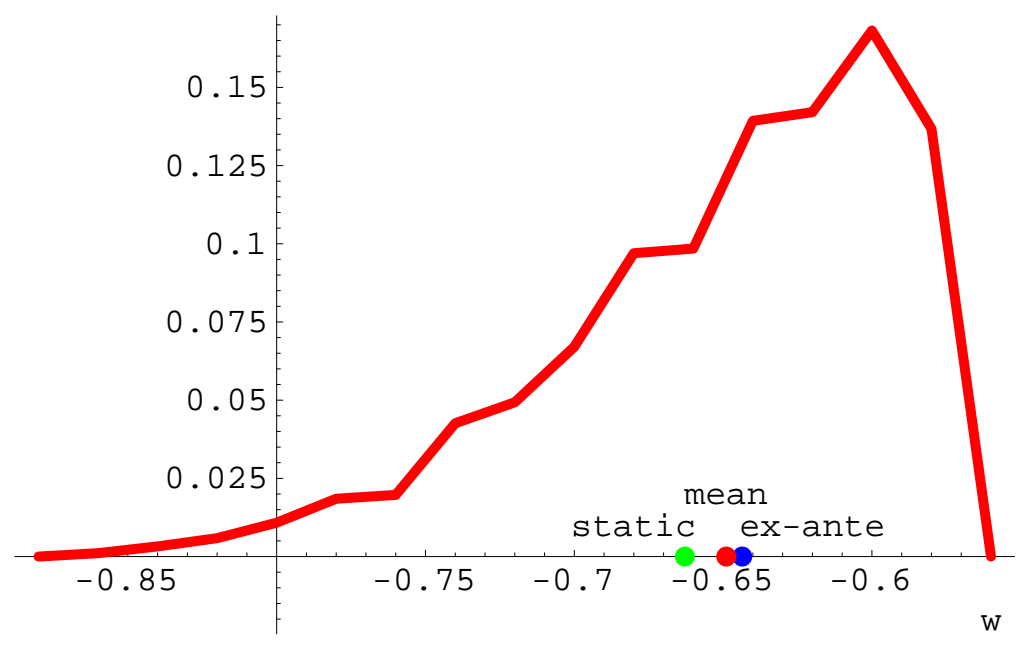

Figure 1: The Distribution of Dynastic Utilities, $\Psi$.

Since the static optimum is a feasible but suboptimal stationary allocation, its value is strictly lower than the mean of $\Psi$. Since the optimal stationary allocation is a feasible allocation when stationarity is not imposed, the mean of $\Psi$ is strictly lower than the utility associated with the optimal ex ante allocation.

For all $w$ in the support of $\Psi, a(w)=1$. (Thus, this function is not graphed.) Figure 2 presents the function $c(w, q)$. Not surprisingly, $c(w, q)$ is increasing in both arguments. Further, for a household receiving the dynastic utility associated with the static optimum, $c(w, q)$ provides less dependence of consumption on current output $q$ than does the static plan. For a household receiving the dynastic utility associated with the ex ante optimum, $c(w, q)$ provides more dependence of consumption on current output than does the ex ante optimum. 


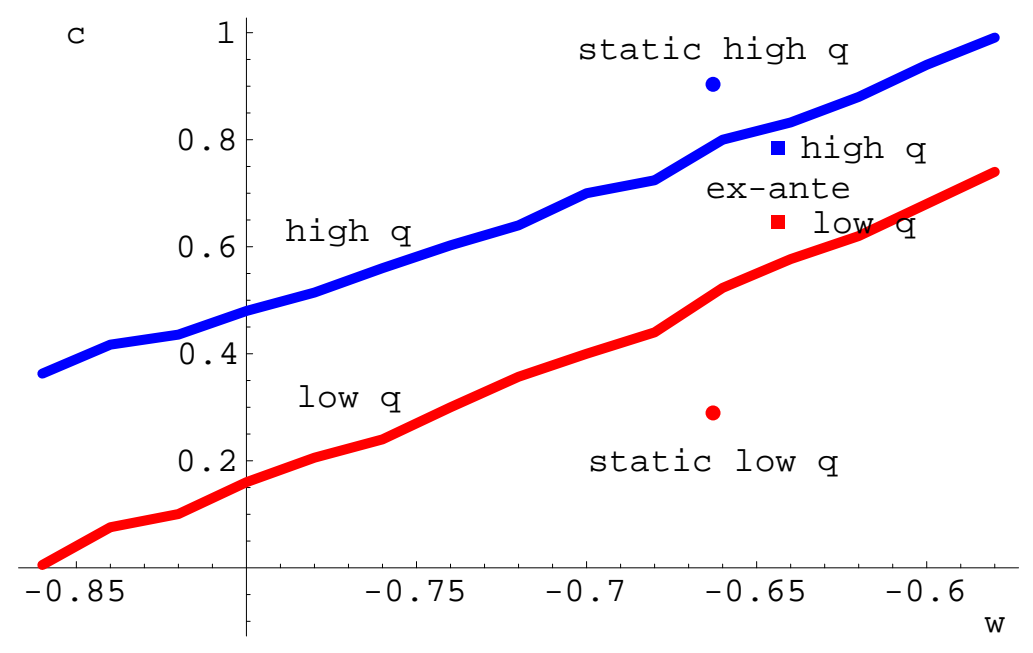

Figure 2: The Consumption Function, $c(w, q)$.

Neither of these characteristics is surprising. That the optimal stationary allocation has less dependence of current consumption on current output comes entirely from the fact that in the static optimum, all incentives must be provided through such dependence, while the optimal stationary allocation allows for incentives to be provided through the function $w^{\prime}(w, q)$ as well. That the optimal stationary allocation has more dependence of current consumption on current output than does the ex ante optimal plan comes from the fact that future generations matter more to society when ranking allocations by mean limiting utility than by ex ante utility. Having a household's consumption depend on its ancestors' output costs society because utility is a convex function of consumption. However, such a dependence helps relax the incentive constraints on parents (Theorem 1). The more future generations enter the objective function of society, however, the costlier such intergenerational dependence, and, thus, the less this manner of providing incentives is used. 


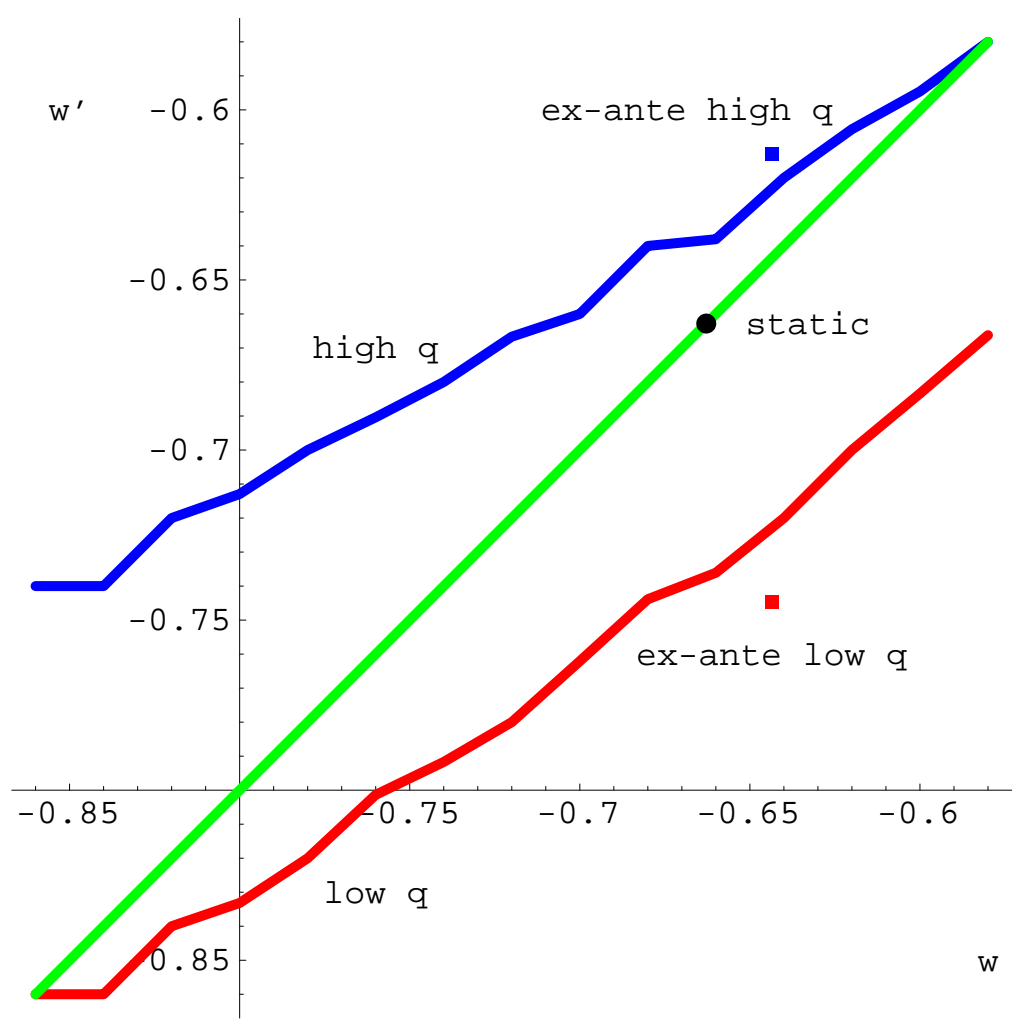

Figure 3: The Utility Transition Function, $w^{\prime}(w, q)$.

Figure 3 presents the transition function $w^{\prime}(w, q)$. Like the function $c(w, q)$, the function $w^{\prime}(w, q)$ is increasing in both arguments. The transition function $w^{\prime}(w, q)$ provides more dependence of future dynastic utility on current output $q$ than does the static optimum, since, by definition, the static optimum allows for no such dependence. For a household receiving the dynastic utility associated with the ex ante optimum, $w^{\prime}(w, q)$ provides less dependence of future dynastic utility on current output than does the ex ante optimum, precisely because $c(w, q)$ is more sensitive to current output than is the ex ante optimum.

\section{Concluding Remarks}

The results here should generalize to environments other than unobserved effort. The idea that at perfect equality the marginal cost of unequal opportunity is second-order but 
the benefits are first-order appears quite general. The result on social mobility should hold for any incentive model in which the resource cost of providing a mean dynastic utility is strictly convex. For instance, an earlier version of this work proves Theorems 1 and 2 for the taste shock model of Atkeson and Lucas (1992). While the need to provide incentives is fundamental here, the particular source of the incentive problem is not. Technically, all that is needed is a binding incentive constraint. With this, both unequal opportunity and social mobility are necessary implications of an efficient, or optimal, societal arrangement. 


\section{References}

Atkeson, Andrew, and Robert E. Lucas, Jr. (1992), "On Efficient Distribution with Private Information," Review of Economic Studies, vol. 59 (3), pp. 427-453.

Farhi, Emmanuel, and Ivan Werning (2005), "Inequality and Social Discounting," manuscript, Massachusetts Institute of Technology.

Freeman, Scott, and Michael A. Sadler (2002), "On the Nonoptimality of Bequeathing Inequality," manuscript, University of Texas at Austin.

Fudenberg, Drew, and Jean Tirole (1991), Game Theory, The MIT Press, Cambridge.

Green, Edward J. (1987), "Lending and the Smoothing of Uninsurable Income," in E. Prescott and N. Wallace (eds.), Contractual Arrangements for Intertemporal Trade, pp. 3-25, University of Minnesota Press, Minneapolis.

Hopenhayn, Hugo A., and Juan Pablo Nicolini (1997) "Optimal Unemployment Insurance," Journal of Political Economy, vol. 105 (2), pp. 412-438.

Khan, Aubhik, and B. Ravikumar (2002), "Enduring Relationships in an Economy with Capital," Working Paper 02-5, Federal Reserve Bank of Philadelphia.

Phelan, Christopher (1994), "Incentives and Aggregate Shocks," Review of Economic Studies, vol. 61 (4), pp. 681-700.

Phelan, Christopher (1995), "Repeated Moral Hazard and One-Sided Commitment," Journal of Economic Theory, vol. 66 (2), pp. 488-506.

Phelan, Christopher (2002), "Inequality and Fairness," Federal Reserve Bank of Minneapolis Quarterly Review, vol. 26 (Spring), pp. 2-11.

Phelan, Christopher, and Robert M. Townsend (1991), "Computing Multi-Period, Information- 
Constrained Optima," Review of Economic Studies, vol. 58 (5), pp. 853-881.

Prescott, Edward C., and Robert M. Townsend (1984), "Pareto Optima and Competitive Equilibria with Adverse Selection and Moral Hazard," Econometrica, vol. 52 (1), pp. $21-45$.

Rawls, John (1971), A Theory of Justice, Belknap Press of Harvard University Press, Cambridge.

Rogerson, William (1985), "Repeated Moral Hazard," Econometrica, vol. 53, pp. 69-76.

Sleet, Chris and Sevin Yeltekin (2005) "Social credibility, social patience and long run inequality, " manuscript, Carnegie Mellon University.

Wang, Cheng (1995), "Dynamic Insurance with Private Information and Balanced Budgets," Review of Economic Studies, vol. 62 (4), pp. 577-596. 


\section{Appendix}

Proof. (Lemma 1): Suppose allocation $\tilde{\zeta}$ solves the primal problem but not the dual problem. Allocation $\tilde{\zeta}$ is in the constraint set of the dual problem with $\operatorname{cost} S(\tilde{\zeta}) \leq 0$. Thus, if it does not solve the dual problem, there exists another allocation $\zeta$ with a cost, $S(\zeta)<0$, satisfying (1), (2) and $\bar{v} \leq \liminf _{T \rightarrow \infty} \frac{1}{T+1} \sum_{t=0}^{T} \int_{V} w d \Psi_{t}(w)$.

For all $t$, define $\Delta_{t}$ such that $\int_{w} \sum_{q} P\left(q_{t} \mid a_{t}\left(w_{t}\right)\right)\left[u^{-1}\left(u\left(c_{t}\left(w_{t}, q_{t}\right)\right)+\Delta_{t}\right)-q_{t}\right] d \Psi_{t}(w)=0$. In words, $\Delta_{t}$ is the constant utility addition (to allocation $\zeta$ ) which causes the resource constraint in period $t$ to hold with equality. Next, let

$$
\begin{aligned}
& \hat{w}_{t+1}\left(w_{t}, q_{t}\right)=w_{t+1}\left(w_{t}-\frac{1-\beta}{\beta^{t}} \sum_{s=t}^{\infty} \beta^{s} \Delta_{s}, q_{t}\right)+\frac{1-\beta}{\beta^{t+1}} \sum_{s=t+1}^{\infty} \beta^{s} \Delta_{s}, \\
& \hat{a}_{t}\left(w_{t}\right)=a_{t}\left(w_{t}-\frac{1-\beta}{\beta^{t}} \sum_{s=t}^{\infty} \beta^{s} \Delta_{s}\right)
\end{aligned}
$$

and $\hat{c}_{t}\left(w_{t}, q_{t}\right)$ be such that

$$
u\left(\hat{c}_{t}\left(w_{t}, q_{t}\right)\right)=u\left(c_{t}\left(w_{t}-\frac{1-\beta}{\beta^{t}} \sum_{s=t}^{\infty} \beta^{s} \Delta_{s}, q_{t}\right)\right)+\Delta_{t}
$$

Finally, for all $(\underline{w}, \bar{w})$ let $\hat{\Psi}_{0}\left([\underline{w}, \bar{w}]=\Psi_{0}\left(\left[\underline{w}-(1-\beta) \sum_{s=0}^{\infty} \beta^{s} \Delta_{s}, \bar{w}-(1-\beta) \sum_{s=0}^{\infty} \beta^{s} \Delta_{s}\right]\right)\right.$. This defines an alternative feasible, incentive-compatible allocation $\hat{\zeta}$ where the resource constraint holds with equality at each date. (Note that for some $t, S_{t}(\zeta)$ may be positive (the resource constraint (3) is violated), in which case this procedure reduces aggregate consumption, and in others, $S_{t}(\zeta)$ is negative (the resource constraint (3) is slack), in which case this procedure increases aggregate consumption.) 
This construction ensures limiting mean utility increases $\left(\right.$ since $\lim \inf _{T \rightarrow \infty} \frac{1}{T+1} \sum_{t=0}^{\infty} \Delta_{t}>$ 0 from $S(\zeta)<0)$. Further, conditions (1), (2), and (3) are satisfied by $\hat{\zeta}$. Thus $\hat{\zeta}$ is in the constraint set of the primal problem, but has a higher primal objective function value than $\tilde{\zeta}$, contradicting the optimality of $\tilde{\zeta}$.

For Lemma 2, it is useful to let allocations be defined as probabilistic. Let a probabilistic allocation by defined as a collection $\zeta=\left(\Psi_{0},\left\{\pi_{t}\left(\cdot \mid w_{t}\right)\right\}_{t=0}^{\infty}\right)$, where $\pi_{t}\left(\cdot \mid w_{t}\right)$ is a probability measure mapping subsets of $A \times Q \times \mathbb{R}_{+} \times V$ to $[0,1]$. In words, $\pi_{t}\left(\cdot \mid w_{t}\right)$ gives the joint probability of a household promised $w_{t}$ dynastic utils being recommended action $a_{t}$, realizing output $q_{t}$, receiving consumption level $c_{t}$, and future utility promise $w_{t+1}$ as in section 5 . As before, the measure $\Psi_{0}$ and the functions $\pi_{t}$ define the sequence of measures over continuation utilities $\left\{\Psi_{t}\right\}_{t=0}^{\infty}$ with means $\left\{\bar{v}_{t}\right\}_{t=0}^{\infty}$.

Proof. (Lemma 2): Suppose there exists $\zeta$ such that $S(\zeta)=S(\xi)-\epsilon$ for $\epsilon>0$. Let $E_{q}=\sum_{q} q P\left(q \mid a_{0}\right)$ where $a_{0}$ is the lowest effort level and let $\bar{v}_{0}$ be the mean of $\Psi_{0}$. Next, define $c^{0}(w)$ such that $w=(1-\beta)\left(u\left(c^{0}(w)\right)-v\left(a_{0}\right)\right)+\beta \bar{v}_{0}$ and $D_{t}(\zeta) \equiv \int_{w} c^{0}(w) d \Psi_{t}(w)-E_{q}-S_{t}(\zeta)$. In words, the function $c^{0}(w)$ is the consumption necessary to keep utility promise $w$, given that from next period on the household will receive utility $\bar{v}_{0}$ and effort is set to $a_{0}$. The function $D_{t}(\zeta)$ is the addition to the cost in period $t$ of replacing the period $t$ allocation with an allocation that delivers $\Psi_{t}$ with efforts set to $a_{0}$ and promises set to $\bar{v}_{0}$. Let $\underline{D} \equiv$ $\liminf _{t \rightarrow \infty} D_{t}(\zeta)$ and suppose $\underline{D}=\infty$. This implies either $S_{t}(\zeta) \rightarrow-\infty$, which contradicts $S(\zeta)=0$, or $\int_{w} c^{0}(w) d \Psi_{t}(w) \rightarrow \infty$. The latter implies either $\bar{v}_{t} \rightarrow-\infty$ or the variance of $\Psi_{t} \rightarrow \infty$, neither of which is compatible with optimality. Thus $\underline{D}<\infty$.

Choose $T$ such that $\frac{1}{T+1} \sum_{t=0}^{T} S_{t}(\zeta)<S(\zeta)+\frac{\epsilon}{2}$, and $\frac{1}{T+1} D_{T}(\zeta)<\frac{\epsilon}{2}$. Let $\zeta^{C}$ equal $\zeta$ for dates $t=0$ to $T-1$, and let $\zeta^{C}$ for date $T$ deliver $\Psi_{T}$ with effort set to $a_{0}$, con- 
sumption levels set to $c^{0}(w)$ and promised utility be a lottery with measure $\Psi_{0}$ independent of $w_{T}$ and $q_{T}$. For all $t>T$, repeat this $T+1$ length cycle. By construction, $S\left(\zeta^{C}\right)<S(\zeta)+\epsilon$. Next, define the stationary allocation $\bar{\xi}=(\bar{\Psi}, \bar{\pi})$ as follows. For all $W \in \mathcal{B}(V), \bar{\Psi}(W)=\frac{1}{T+1} \sum_{t=0}^{T} \Psi_{t}^{C}(W)$ and for all $W \in \mathcal{B}(V)$, and $Z \in \mathcal{B}\left(A \times Q \times \mathbb{R}_{+} \times V\right)$, $\int_{w} \bar{\pi}(Z \mid w) d \bar{\Psi}(w)=\left(\sum_{t=0}^{T} \Psi_{t}^{C}(w)\right)^{-1} \sum_{t=0}^{T} \int_{w} \pi_{t}^{C}(Z \mid w) d \Psi_{t}^{C}(w)$. (If $\sum_{t=0}^{T} \Psi_{t}^{C}(w)=0$, then $\bar{\pi}$ can be defined as anything.) This construction ensures $\int_{w} w d \bar{\Psi}(w)=\frac{1}{T+1} \sum_{t=0}^{T} \int_{w} w \Psi_{t}^{C}(w)$, and thus the stationary allocation $\bar{\xi}$ delivers the same average utility as the cycle $\zeta^{C}$. Second, this construction ensures $\int_{w} \int_{a, q, c, w^{\prime}}(c-q) d \bar{\pi}\left(a, q, c, w^{\prime}\right) d \bar{\Psi}(w)=\frac{1}{T+1} \sum_{t=0}^{T} \int_{w} \int_{a, q, c, w^{\prime}}(c-$ q) $\pi_{t}^{C}\left(a, q, c, w^{\prime}\right) d \Psi_{t}^{C}(w)$, and thus the stationary allocation $\bar{\xi}$ has the same cost as the cycle $\zeta^{C}$. Since $S(\bar{\xi})<S(\zeta)+\epsilon<S(\xi)$, the allocation $\xi$ could not have solved the stationary dual problem.

Proof. (Lemma 3): Consider a solution to the stationary primal problem $\xi^{*}$ such that

$$
\int_{V} \sum_{q} P\left(q \mid a^{*}(w)\right)\left[c^{*}(w, q)-q\right] d \Psi^{*}(w)<0
$$

For a given $\epsilon>0$, construct an alternative allocation $\xi$ as follows. First, for all intervals $(-\infty, w]$, let $\Psi((-\infty, w+\epsilon])=\Psi^{*}((-\infty, w])$. This ensures that the objective function increases by $\epsilon$. Next, let $a(w+\epsilon)=a^{*}(w)$. This ensures that aggregate production is unchanged. Finally, let $w^{\prime}(w+\epsilon, q)=w^{\prime *}(w, q)+\epsilon, c(w+\epsilon, q)=c^{*}(w, q)$ if $q \neq \bar{q}$, and $c(w+\epsilon, \bar{q})$ be such that

$$
u(c(w+\epsilon, \bar{q}))=u\left(c^{*}(w, \bar{q})\right)+\frac{\epsilon}{P\left(\bar{q} \mid a^{*}(w)\right)} .
$$


In words, a household promised $w$ utils under allocation $\xi^{*}$ is delivered $w+\epsilon$ utils under allocation $\xi$ by increasing all continuation utility promises by $\epsilon$ and increasing the utility payment if output $\bar{q}$ occurs by $\epsilon / P\left(\bar{q} \mid a^{*}(w)\right)$. This construction ensures that stationarity (4) and promise-keeping (5) are maintained.

This leaves the incentive-compatibility constraints (6) and the resource-feasibility condition (7) to be met. For a given utility point $w+\epsilon$ and $\hat{a}<a$, the incentive constraint for allocation $\xi$ is that

$$
w+\epsilon \geq \sum_{q} P(q \mid \hat{a})\left((1-\beta)[u(c(w+\epsilon, q))-v(\hat{a})]+\beta w^{\prime}(w+\epsilon, q)\right) .
$$

From the fact that $\xi^{*}$ is incentive-compatible, we know that

$$
w=\sum_{q} P(q \mid \hat{a})\left((1-\beta)\left[u\left(c^{*}(w, q)\right)-v(\hat{a})\right]+\beta w^{\prime *}(w, q)\right)+\Delta,
$$

where $\Delta \geq 0$ is the amount by which the incentive constraint is slack. Subtracting, side by side, expression (21) from expression (20) and using the definition of $\xi$ delivers that

$$
\epsilon \geq(1-\beta) \frac{P(\bar{q} \mid \hat{a})}{P\left(\bar{q} \mid a^{*}(w)\right)} \epsilon+\beta \epsilon-\Delta
$$

This holds because $P(\bar{q} \mid \hat{a}) / P\left(\bar{q} \mid a^{*}(w)\right)<1$ and $\Delta \geq 0$. Thus, $\xi$ is incentive-compatible.

Finally, the fact that $\int_{V} \sum_{q} P\left(q \mid a^{*}(w)\right)\left[c^{*}(w, q)-q\right] d \Psi^{*}(w)<0$ implies that there exists an $\epsilon>0$ for which equation (7) is satisfied, contradicting the optimality of $\xi^{*}$.

Next, consider a solution to the stationary dual problem $\xi^{*}$ such that $\int_{V} w d \Psi^{*}(w)=$ $\bar{v}+\epsilon$ for $\epsilon>0$. Construct an alternative allocation exactly as in the previous paragraphs, but 
instead of adding consumption when output $\bar{q}$ occurs, subtract consumption when output $\underline{q}$ occurs. This lowers both cost (the objective function of the stationary dual problem) and mean utility (which is slack). Since $\epsilon>0$, this contradicts the optimality of $\xi^{*}$.

Proof. (Lemma 4): If $\xi^{*}$ solves either the stationary primal or stationary dual problem, it satisfies (4)-(6) immediately. Suppose $\xi^{*}$ solves the stationary primal problem and another stationary allocation $\xi$ satisfying (4)-(6) and (9) has a negative value of the stationary dual objective function (8). Such a plan is in the constraint set of the stationary primal problem since it satisfies (4)-(6) immediately and satisfies (7) with slack. Stationary allocation $\xi$ has a weakly higher stationary primal objective function value (since it satisfies (9)) and the resource constraint (7) does not bind, contradicting Lemma 3. Thus, if $\xi^{*}$ solves the stationary primal problem with objective value $v$, it solves the stationary primal problem with $C(\bar{v})=0$.

Next, suppose a stationary allocation $\xi^{*}$ solves the stationary dual problem with $C(\bar{v})=0$. Suppose another stationary allocation $\xi$ satisfying $(4)-(7)$ has $\int_{V} w d \Psi(w)>\bar{v}$. Such a plan is in the constraint set of the stationary dual problem since it satisfies (4)(6) immediately and satisfies (9) with slack. Stationary allocation $\xi$ has a weakly higher stationary dual objective function value (since it satisfies (7)) and (9) does not bind, contradicting Lemma 3. Thus, if $\xi^{*}$ solves the stationary dual problem with $C(\bar{v})=0$, it solves the stationary primal problem with objective value $\bar{v}$.

Proof. (Lemma 5): To begin, note $V=(-\infty, 0)$. Next, let $\xi_{-1}=\left(\Psi_{-1}, a_{-1}(w), c_{-1}(w, q)\right.$, $\left.w_{-1}^{\prime}(w, q)\right)$ solve (11) subject to (4)-(6) and (9) for $\bar{v}=-1$. Next, fix $\Delta>0$, and define $\xi_{\Delta}$ by scaling $\xi_{-1}$ as follows: First, for all $\{\underline{w}, \bar{w}\} \in \mathbb{R}_{-}^{2}$, let $\Psi_{\Delta}([\underline{w}, \bar{w}])=\Psi_{-1}([\underline{w} / \Delta, \bar{w} / \Delta])$. Next, let $a_{\Delta}(w)=a_{-1}(w / \Delta), c_{\Delta}(w, q)=c_{-1}(w / \Delta, q)-\log (\Delta) / \gamma$, and $w_{\Delta}^{\prime}(w, q)=w_{-1}^{\prime}(w / \Delta, q) \Delta$. By construction, $\xi_{\Delta}$ satisfies (4). Next, consider the incentive constraint for a given $w<0$ 
and $\hat{a}<a_{\Delta}(w)$, that

$$
\begin{aligned}
& \sum_{q} P\left(q \mid a_{\Delta}(w)\right)\left\{(1-\beta)\left[-\exp \left(-\gamma\left[c_{\Delta}(w, q)-v\left(a_{\Delta}(w)\right]\right)\right]+\beta w_{\Delta}^{\prime}(w, q)\right\}\right. \\
\geq & \sum_{q} P(q \mid \hat{a})\left\{(1-\beta)\left[-\exp \left(-\gamma\left[c_{\Delta}(w, q)-v(\hat{a})\right]\right)\right]+\beta w_{\Delta}^{\prime}(w, q)\right\} .
\end{aligned}
$$

With the definition of $\xi_{\Delta}$ substituting into this, it simplifies to

$$
\begin{aligned}
& \sum_{q} P\left(q \mid a_{-1}(w)\right)\left\{(1-\beta)\left[-\exp \left(-\gamma\left[c_{-1}(w / \Delta, q)-v\left(a_{-1}(w / \Delta)\right)\right]\right)\right]+\beta w_{-1}^{\prime}(w / \Delta, q)\right\} \\
\geq & \left.\sum_{q} P(q \mid \hat{a})(1-\beta)\left[-\exp \left(-\gamma\left[c_{-1}(w / \Delta, q)-v(\hat{a})\right]\right)\right)\right]+\beta w_{-1}^{\prime}(w / \Delta, q),
\end{aligned}
$$

which holds since $\xi_{-1}$ is incentive-compatible, or satisfies (6). Next, confirm the promisekeeping constraint (5) by noting that

$$
\begin{aligned}
& \sum_{q} P\left(q \mid a_{\Delta}(w)\right)\left\{(1-\beta)\left[-\exp \left(-\gamma\left[c_{\Delta}(w, q)-v\left(a_{\Delta}(w)\right)\right]\right)\right]+\beta w_{\Delta}^{\prime}(w, q)\right\} \\
= & \Delta \sum_{q} P\left(q \mid a_{-1}(w)\right)\left\{(1-\beta)\left[-\exp \left(-\gamma\left[c_{-1}(w / \Delta, q)-v\left(a_{-1}(w)\right)\right]\right)\right]+\beta w_{-1}^{\prime}(w / \Delta, q)\right\} \\
= & \Delta w / \Delta=w .
\end{aligned}
$$

Further, $\xi_{\Delta}$ satisfies (9) for $\bar{v}=-\Delta$ since

$$
\int_{V} w d \Psi_{\Delta}(w)=\int_{V} w \Delta d \Psi_{-1}(w)=\Delta \int_{V} w d \Psi_{-1}(w)=-\Delta
$$

Thus, $\xi_{\Delta}$ is in the constraint set of the dual problem for $\bar{v}=-\Delta$. The resources consumed 
by $\xi_{\Delta}$ can be expressed as

$$
\begin{aligned}
& \int_{V} \sum_{q} P\left(q \mid a_{\Delta}(w)\right)\left[c_{\Delta}(w, q)-q\right] d \Psi_{\Delta}(w) \\
= & \int_{V} \sum_{q} P\left(q \mid a_{\Delta}(w \Delta)\right)\left[c_{\Delta}(w \Delta, q)-q\right] d \Psi_{-1}(w) \\
= & \int_{V} \sum_{q} P\left(q \mid a_{-1}(w)\right)\left[c_{-1}(w, q)-\log (\Delta) / \gamma-q\right] d \Psi_{-1}(w) \\
= & -\log (-v) / \gamma+\int_{V} \sum_{q} P\left(q \mid a_{-1}(w)\right)\left[c_{-1}(w, q)-q\right] d \Psi_{-1}(w) \\
= & -\log (-v) / \gamma+C(1) .
\end{aligned}
$$

Next, suppose that there exists a plan $\xi^{*}$ satisfying (4)-(6) and (9) for $\bar{v}=-\Delta$, which has resource $\operatorname{cost} C^{*}<-\log (-\bar{v}) / \gamma+C(-1)$. Here, let $\delta=-1 / \Delta$, and define $\xi_{\delta}$ by scaling $\xi^{*}$ by $\delta$ as above. The same arguments as above establish that $\xi_{\delta}$ satisfies stationarity, incentive-compatibility (6), and promise-keeping (5). Further,

$$
\int_{V} w d \Psi_{\delta}(w)=\int_{V} w / \delta d \Psi^{*}(w)=(1 / \delta) \int_{V} w d \Psi^{*}(w)=-1
$$

and thus $\xi_{\delta}$ satisfies (9) for $\bar{v}=-1$. The resource cost of $\xi_{\delta}$ is, then,

$$
\begin{aligned}
& \int_{V} \sum_{q} P\left(q \mid a_{\delta}(w)\right)\left[c_{\delta}(w, q)-q\right] d \Psi_{\delta}(w) \\
= & \int_{V} \sum_{q} P\left(q \mid a_{\delta}(w \delta)\right)\left[c_{\delta}(w \delta, q)-q\right] d \Psi^{*}(w) \\
= & \int_{V} \sum_{q} P\left(q \mid a^{*}(w)\right)\left[c^{*}(w, q)-\log (\delta) / \gamma-q\right] d \Psi^{*}(w) \\
= & -\log (-1 / \bar{v}) / \gamma+\int_{V} \sum_{q} P\left(q \mid a^{*}(w)\right)\left[c^{*}(w, q)-q\right] d \Psi^{*}(w) \\
= & \log (-\bar{v}) / \gamma+C^{*}<\log (-\bar{v}) / \gamma-\log (-\bar{v}) / \gamma+C(-1)=C(-1),
\end{aligned}
$$


which contradicts optimality of $\xi_{-1}$.

Proof. (Lemma 6): The proof of Lemma 6 proceeds in the same way as that of Lemma 5. For $\gamma>0, \xi_{1}=\left\{\Psi_{1}, k_{1}(w), a_{1}(w), c_{1}(w, q), w_{1}^{\prime}(w, q)\right\}$ is defined as the optimal allocation delivering $\bar{v}=1$. Then, for $\Delta>0$, a new allocation $\xi_{\Delta}$ is defined such that for all $[\underline{w}, \bar{w}] \in \mathbb{R}_{+}^{2}$, $\Psi_{\Delta}([\underline{w}, \bar{w}])=\Psi_{1}([\underline{w} / \Delta, \bar{w} / \Delta]), k_{\Delta}(w)=k_{1}(w / \Delta) \Delta^{1 / \gamma}, a_{\Delta}(w)=a_{1}(w / \Delta), c_{\Delta}(w, q)=$ $c_{1}(w / \Delta, q) \Delta^{1 / \gamma}$, and $w_{\Delta}^{\prime}(w, q)=w_{1}^{\prime}(w / \Delta, q) \Delta$. I can show that $\xi_{\Delta}$ is satisfied for $(4)-(6)$ and (9) for $\bar{v}=\Delta$. Further, if any other plan had a lower value for the dual objective function (12), it could be used to generate a lower cost plan for delivering $\bar{v}=1$. Then $c_{\Delta}(w, q)=c_{1}(w / \Delta, q) \Delta^{1 / \gamma}$, and $\bar{v}=\Delta$ delivers $C(\bar{v})=\bar{v}^{1 / \gamma} C(1)$.

For $\gamma<0, \xi_{-1}=\left\{\Psi_{-1}, k_{-1}(w), a_{-1}(w), c_{-1}(w, q), w_{-1}^{\prime}(w, q)\right\}$ is defined as the optimal allocation delivering $\bar{v}=-1$. Then, for $\Delta>0$, a new allocation $\xi_{\Delta}$ is defined such that for all $[\underline{w}, \bar{w}] \in \mathbb{R}_{-}^{2}, \Psi_{\Delta}([\underline{w}, \bar{w}])=\Psi_{-1}([\underline{w} / \Delta, \bar{w} / \Delta]), k_{\Delta}(w)=k_{-1}(w / \Delta) \Delta^{1 / \gamma}, a_{\Delta}(w)=a_{-1}(w / \Delta)$, $c_{\Delta}(w, q)=c_{-1}(w / \Delta, q) \Delta^{1 / \gamma}$, and $w_{\Delta}^{\prime}(w, q)=w_{-1}^{\prime}(w / \Delta, q) \Delta$, and the argument proceeds unaltered. Then $c_{\Delta}(w, q)=c_{-1}(w / \Delta, q) \Delta^{1 / \gamma}$, and $\bar{v}=-\Delta$ delivers $C(\bar{v})=(-\bar{v})^{1 / \gamma} C(-1)$.

Finally, for $\gamma=0$, or $U(c, a, k)=\log (c-k v(a))$, the reference allocation $\xi_{0}=$ $\left\{\Psi_{0}, k_{0}(w), a_{0}(w), c_{0}(w, q), w_{0}^{\prime}(w, q)\right\}$ is defined as the optimal allocation delivering $\bar{v}=0$. Then, for $\Delta \in \mathbb{R}$, a new allocation $\xi_{\Delta}$ is defined such that for all $[\underline{w}, \bar{w}] \in \mathbb{R}^{2}, \Psi_{\Delta}([\underline{w}, \bar{w}])=$ $\Psi_{0}([\underline{w}+\Delta, \bar{w}+\Delta]), k_{\Delta}(w)=k_{0}(w-\Delta) \exp (\Delta), a_{\Delta}(w)=a_{0}(w-\Delta), c_{\Delta}(w, q)=c_{0}(w-$ $\Delta, q) \exp (\Delta)$, and $w_{\Delta}^{\prime}(w, q)=w_{0}^{\prime}(w-\Delta, q)+\Delta$, and the argument proceeds unaltered. Then $c_{\Delta}(w, q)=c_{0}(w-\Delta, q) \exp (\Delta)$, and $\bar{v}=\Delta$ delivers $C(\bar{v})=\exp (\bar{v}) C(0)$ 